\title{
A detailed examination of mediation in PA learning*
}

\author{
JOHN C. YUILLE† \\ University of British Columbia, Vancouver, British Columbia, Canada
}

\begin{abstract}
This paper reports a single experiment in which verbal and imaginal mediators were compared in paired-associate learning. Pair concreteness and delay of recall (immediate vs 1 -week delay) were also manipulated. The dependent variables included the latencies of mediator discovery and mediator production, response recall, mediator recall, and recall latency. A variety of differences between verbal and imaginal codes were found in the dependent measures. In addition, a profound effect of immediate recall on subsequent recall was discovered. The results are discussed in terms of the differences between the two modalities of memory.
\end{abstract}

The past decade of research hàs witnessed the establishment of the importance of mediation processes in associative learning. Beginning with the work of Bugelski (1962), a large number of studies have shown a strong, positive relationship between reported mediation and recall performance (e.g., Kiess \& Montague, 1965; Paivio, Yuille, \& Smythe, 1966). Furthermore, the complexity of reported mediators (e.g., Martin, Cox, \& Boersma, 1965) and mediator recall (e.g., Montague, Adams, \& Kiess, 1966) are both correlated with response recall. Of course, a basic interpretive problem exists with these data in that no cause and effect relationship can be established between mediator recall and response recall Nonetheless, the correlational evidence is substantial (and consistent with the result that most researchers in the field accept a major role for mediation processes in associative learning).

More direct support for the importance of mediators in learning is found in research efforts to manipulate mediation processes. Instructions to mediate result in superior performance over rote memorization instructions, or no special instructions, whether these manipulations are between Ss (Yuille \& Paivio, 1968) or within Ss (Schnorr \& Atkinson, 1969).

The acceptance of a central role for mediation has resulted in more recent studies concentrating on the variety of mediators and their relationship to the characteristics of the to-be-learned material. Most of this research has been concerned with the comparison of verbal and nonverbal mediators (for summaries of this work, see Bower, 1972; Paivio, 1971). These research efforts have established that $S$ may use either a verbal mediator or an image (or both) to code a paired associate. Which type of mediator $\mathrm{S}$ will employ for a

\footnotetext{
*This research was supported by grants from the National Research Council of Canada (AO 327) and the University of British Columbia. The author gratefully acknowledges the assistance of Richard Hardisty in the collection and analysis of the data. An earlier version of this paper was read at the annual meeting of the Canadian Psychological Association, 1972.

$\uparrow$ Reprint requests should be sent to Dr. John C. Yuille, Department of Psychology, University of British Columbia Vancouver 8, British Columbia, Canada.
}

particular pair depends on instructions, the characteristics of the pair, as well as S's coding habits.

Instructions to employ a particular type of mediator appear to control tie coding strategy that $S$ uses as long as that strategy is effective in facilitating his recall (Paivio \& Yuille, 1969). Alternatively, pair concreteness affects mediation in that Ss are more likely to report using nonverbal imagery to mediate concrete material and verbal devices to mediate more abstract pairs (Paivio et al, 1966). Paivio (1971) has proposed that this relationship between mediator type and pair concreteness holds for the entire dimension of stimulus concreteness, from objects through pictures and concrete nouns to abstract words.

The major consequence of this type of research has been the growth of a general framework for the interpretation of associative learning. The fundamental assumption of this approach is that mediation and learning are virtually synonymous. Rote (nonmediated) ${ }^{1}$ learning is accepted as one method of storing information, but it is assumed that the learning of meaningful material generally involves mediation. When $S$ mediates a pair of items, he actively seeks an association or mnemonic or interaction of the items. The discovery of a mediator for a pair determines the nature of the storage for that particular pair. For example, if $S$ is presented a pair of pictures to associate, he may generate a nonverbal interaction of the pictures, and the storage would be visual. However, S may label the pictures with a consequent verbal storage. In other words, mediator discovery for a pair is tantamount to storage of that pair (for a more detailed discussion of learning stages, see Humphreys \& Yuille, 1972).

The relationship between the type of mediator and pair concreteness exemplifies the effect of mediation on storage. As both members of a pair become more concrete, the likelihood that $S$ will use an imaginal code increases, and the pair is likely to be stored nonverbally. Alternatively, the probability of verbal storage increases as pairs become more abstract. If $\mathrm{S}$ is not free to choose a code, for example, if instructions require him to generate a particular mediator type, the effect of concreteness on mediation will be reflected in the 
latencies of mediator discovery. Verbal material. regardless of concreteness. can he coded rapidly by verbal mediators, while images require longer to generate for abstract than for concrete word pairs (e.g.. Yuille \& Paivio, 1968).

To summarize these ideas about storage of pairs: (1) storage predominantly involves mediation: (2) at least two types of mediation exist, verbal and imaginal; and (3) with pairs of words the discovery of imaginal, but not verbal, mediators is affected by word concreteness.

The above general description of mediation processes during storage would find general agreement among investigators in the field. However, less unanimity is found when the retrieval stage is discussed. The problem centers on the reason for superior recall of concrete as opposed to abstract material. Since the relationship between type of mediation and concreteness is firmly established, the higher recall of concrete material requires explanation in terms of mediation. Paivio (1971) has presented a two-process interpretation of the concreteness effect on recall. He hypothesizes that concrete material is likely to be coded both verbally and nonverbally, while the abstract pairs are coded only verbally. As two codes are better than one, the concrete material is more easily or more likely to be retrieved. Paivio (1971) has cited some inferential support for this model, but no direct supporting evidence has been reported. Furthermore, work on long-term visual memory (e.g., Shepard, 1967) suggests that visual memory may be superior to verbal memory. This hypothesis has been advanced by Bower (1972) and Yuille and Paivio (1969) to interpret the concreteness effect: the more concrete a pair, the more likely it will be visually coded, and visual coding is superior to verbal coding. To date, no reason for the superiority of coding in the visual system has been advanced. ${ }^{2}$

Experimental attempts at comparing the efficiency of verbal and nonverbal codes has not resulted in support for either of the above interpretations. These studies have attempted to control the type of mediation via instructions and have resulted generally in no recall differences between Ss instructed to use verbal mediators and those asked to use images (e.g., Yuille \& Paivio, 1969). This type of research has two basic problems. First, Ss tend to return to habitual coding methods unless a strong instructional set is used (cf. Paivio \& Yuille, 1969). Second, for most of this work, Ss were asked to verbally describe their imaginal, as well as verbal, mediators. Of course, this procedure encouraged dual coding and did not separate mediator types. Paivio and Foth (1970) overcame the latter problem by having Ss draw their images and write out verbal mediators. The same procedure will be employed in the present study.

The purpose of the present study was to examine in detail the nature of the two types of mediation. The procedure required each $S$ to generate verbal mediators for some pairs and imaginal mediators for others. The concreteness of buhth the stimulus and response members of pairs was varicd by employing concrete and abstract nouns. While these manipulations were not novel, the combination of dependent measures was. The latencies of mediator discovery were recorded. as well as the time required to produce each mediator. In addition, Ss were asked to recill their mediators along with response noun recall, and the recall latencies were measured. Finally, recall was obtained both immediately after the study trial and 1 week later, so that the long-term effects of the two mediator ty pes could be assessed.

While the likelihood of dual coding was reduced in this study by having Ss write their verbal mediators and draw their images, the problem of encouraging $S$ to follow the instructions remained. Since image discovery requires longer for abstract than for concrete pairs, the presentation time for each pair is a critical factor in determining whether $S$ can generate a nonverbal link. In order to preclude presentation time effecting the discovery of the appropriate type of mediation, Ss were permitted to pace themselves during the study trial.

The present experiment permitted a detailed examination of the discovery and production of the two types of mediators as a function of pair concreteness. In addition, the relationships between mediator discovery and production and mediator and response recall were investigated. As noted above, both immediate and delayed recall were assessed. It was decided to include an additional variable: immediate recall vs no immediate recall. This permitted an examination of the effects of immediate recall, or the lack of it, on delayed recall performance. A number of researchers in the field of education have reported that memory tests immediately following learning improve later memory for the material (e.g., Bruning, 1968; Frase, 1967; Gates, 1917; Rothkopf, 1966; Rothkopf \& Bisbicos, 1967). However, the majority of this work has concentrated on prose learning and the use of exam-like memory tests (although see Montague, 1972). The present study presented an excellent opportunity to examine immediate recall effects in a verbal learning paradigm. Consequently, for exploratory purposes, half of the Ss were tested both immediately after and 1 week after the study trial. The remaining Ss were given only the delayed recall. It emerged as a most fortunate turn of events that this variable was included in the design.

\section{METHOD}

\section{Subjects $^{3}$}

The Ss were 32 undergraduate student volunteers ( 24 females, 8 males).

\section{Design and Materials}

Each S was presented a 40-pair list composed of 10 pairs each of concrete-concrete (CC), concrete-abstract (CA), abstract-concrete (AC), and abstract-abstract (AA) nouns. These pairs represent three levels of pair concreteness (both members 
concrete, one noun concrete, both nouns abstract) with CA and $A C$ pairs permitting a comparison of the cueing effects during recall of concrete and abstract stimuli. For half (5) of each of these pair types, $S$ was required to generate a verbal mediator, i.e., a phrase or sentence linking the two members of a pair. For the remaining pairs of each of the four types, $S$ was to discover an image for each pair, i.e., a visual representation linking the members of a pair. Thus, the four types of pairs and the two types of mediator were both manipulated within Ss. The between-Ss variable was the number of recall trials (one vs two). One-half of the Ss were given one recall trial, 7 days after the study trial (delay only condition). The other $16 \mathrm{Ss}$ were given two recall trials (immediate condition), one immediately after the study trial and a second recall trial 7 days after the study trial.

Two separate lists of 40 pairs of nouns each were constructed for use in the learning task. The 160 nouns ( 80 concrete, 80 abstract) were selected from the pool of nouns supplied by Paivio, Yuille, and Madigan (1968). The nouns were paired to form 20 pairs each of $\mathrm{CC}, \mathrm{CA}, \mathrm{AC}$, and AA pairs, with half of these (10 of each type) making up List $A$ and the remainder List B. Each pair of nouns, as well as each stimulus noun alone, was typed in capital letters and mounted as a $35-\mathrm{mm}$ slide.

\section{Apparatus}

The study and recall trial materials were projected via a Kodak Carousel projector onto a blank wall approximately $1.5 \mathrm{~m}$ from S. A clock was wired to the projector so that when a slide was exposed, the clock started. A button in front of S stopped the clock and removed the slide. This clock recorded the latency of mediator discovery. A second clock was started when the first clock stopped and was itself stopped by a second button in front of $\mathrm{S}$. This clock recorded the mediator production time. The final piece of equipment consisted of two small signs in front of $S$ (at desk level). One sign read "Picture" and the other "Sentence." Each sign could be illuminated to indicate the appropriate type of mediator $S$ was to generate for a particular pair.

\section{Procedure}

Each $S$ was tested individually in a small room. The study session began by reading $S$ a set of instructions which described imaginal and verbal mediation, giving examples of both. Four practice pairs were presented to $S$, followed by one of the two 40-pair lists, one pair at a time. Accompanying the display of each pair, the picture or sentence sign in front of $S$ was illuminated to indicate which mediator type he was to generate for that pair. He pressed a switch when he had discovered the appropriate mediator, then drew it if it was a picture or wrote it if it was a sentence. A second switch was pressed when the production of the mediator was completed. This sequence of events was entirely self-paced.

After the completion of the study trial, those $S s$ in the immediate condition were given a recall trial, the other Ss were dismissed. Every $S$ returned 1 week later for a recall trial. The recall procedure was the same for both the immediate and the delayed recall trials. The 40 stimulus nouns were presented one at a time. When $S$ recalled the response, he pressed a switch, then wrote out the response, and reproduced the mediator he had generated during the study trial for that pair. He was encouraged to guess or to press the switch when he felt that he could not retrieve the response (or mediator). The recall trial was also self-paced.

It should be noted that in addition to two lists, two orders of presentation (on study and recall trials) of each list were employed. Within each presentation order, two arrangements of mediator types (i.e., which mediator type was required for a particular pair) were used. Thus, two $S$ s in each group received one of the eight combinations of list, presentation order, and mediator arrangement.

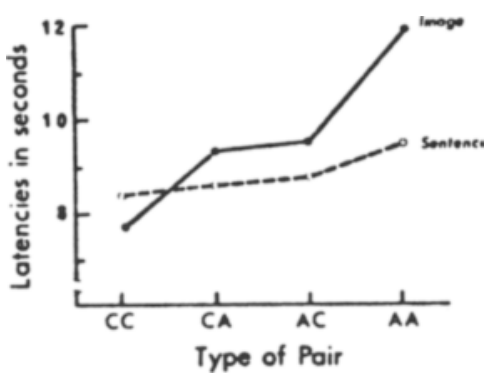

Fig. 1. Mean mediator discovery latencies.

\section{RESULTS AND DISCUSSION}

For each of the measures discussed below, initial analyses included ist and order effects as variables. These factors were not associated with any significant main effects or interactions. Consequently, all the results reported below are collapsed over these variables. All the post hoc analyses employed involve either tests of simple main effects or the Newman-Keuls procedure.

The first dependent measure to be presented is the mediator discovery latencies. For each $\mathrm{S}$, a median latency was calculated for the five pairs of each pair type for which images and sentences were reported. Figure 1 presents the means of these discovery latencies. Note that the discovery time for sentences does not vary as a function of pair type, while images require longer discovery times as pairs become more abstract. An analysis of variance confirms this pattern: the main effects of pair type $[F(3,90)=10.54, p<.01]$ and type of mediator $[F(1,30)=4.70, p<.05]$ were qualified by an interaction of the variables $[F(3,90)=5.29, p<.01]$. Post hoc tests revealed no differences among pair types for sentence discovery. For image discovery, CC pairs required significantly less time than did $\mathrm{CA}$ or $\mathrm{AC}$ pairs, and $\mathrm{AA}$ pairs required significantly longer than did the other pair types.

These results simply replicate the pattern of earlier findings (e.g., Paivio \& Foth, 1970; Yuille \& Paivio, 1967, 1968). These latencies are longer than those reported in the earlier studies. This is a consequence of the self-paced nature of the present experiment, while earlier researchers gave Ss a maximum time limit (e.g., $15 \mathrm{sec})$ to discover a mediator for each pair. Nonetheless, the present results confirm that verbal coding is unaffected by the concreteness of the material, while imaginal codes become increasingly more difficult to discover as pairs become more abstract.

The production latencies represent more novel data, in that the time required to draw or write a mediator has not been previously measured. As with the discovery latencies, a median was calculated for the five pairs in each condition. The means of these latencies are portrayed in Fig. 2. Note the similarity of the pattern to that of Fig. 1, except that a consistent difference between sentence and image production time is apparent. The analysis of variance confirmed this in a 


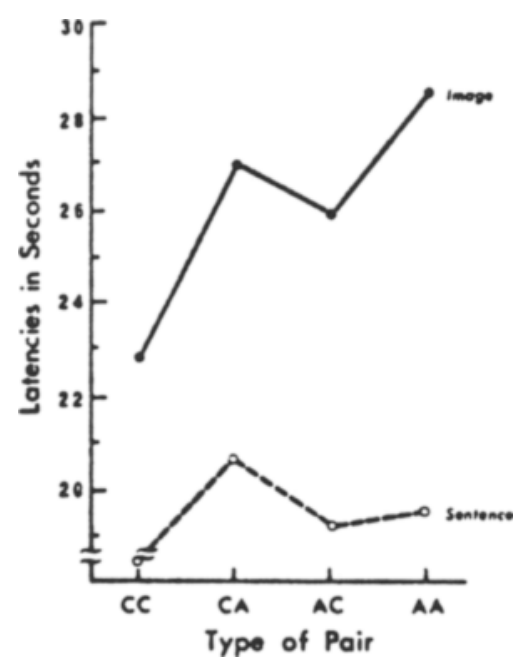

Fig. 2. Mean mediator production latencies.

significant main effect of mediator type $[F(1,30)=$ $30.43, p<.01]$. The main effect of pair type $[F(3,90)=$ $10.55, \mathrm{p}<.01]$ was qualified by the significant interaction $[F(3,90)=4.59, \mathrm{p}<.05]$. The post hoc tests showed that the production time for sentences did not vary as a function of concreteness. However, image production required significantly longer for $\mathrm{CA}$ and $\mathrm{AC}$ pairs than for $\mathrm{CC}$ pairs. Furthermore, drawing images for AA pairs took significantly longer than for the other three pair types.

The lack of any relationship between sentence production time and concreteness is not surprising. It seems obvious that once a sentence has been discovered, it should take the same time to write regardless of the pair type. More unexpected was the finding that the time required to draw an image varied as a function of pair type. It is possible that Ss had not discovered an image when they pressed the first switch. Thus, part of the production latency would reflect discovery latency, which is known to be affected by concreteness. However, it was not apparent to $E$ that Ss took longer to begin drawing images after pressing the first button for abstract as compared to concrete pairs. This observation does not rule out the discovery-production confounding problem, but it certainly qualifies it.

A more plausible explanation of the variability of image production is that image mediators differ as a function of pair type. One possibility is that more complex images are generated for more abstract pairs. An objective test of this possibility proved impossible. However, two judges examined the image mediators for $\mathrm{CC}$ and AA pairs. They classified the drawings for AA pairs as more complex than those produced for CC pairs. The former were more complex in that more objects were included in the drawings and more features of each object were detailed. This difference in detail would explain the differences in production time.

A difference in the detail or complexity of image mediators as a function of concreteness might be expected in terms of referent specificity. Usually only a few visual characteristics are necessary to represent the referent of a concrete noun (e.g., circle). However, since the association between a picture referent and an abstract noun is less direct (e.g., mercy), more detail probably has to be included in these mediators to assume remembering the correct noun. This proposed image variability, as a function of word concreteness, could be the locus for the differential retrieval of images by concrete and abstract stimuli obtained in the present and previous studies.

Combining the discovery and production latencies reveals that Ss required approximately $28 \mathrm{sec}$ to discover and produce a sentence regardless of pair type. The discovery and production of images required $30 \mathrm{sec}$ for $\mathrm{CC}$ pairs, $35 \mathrm{sec}$ for $\mathrm{CA}$ and $\mathrm{AC}$ pairs, and $40 \mathrm{sec}$ for $\mathrm{AA}$ pairs.

It should be noted that no differences in either discovery or production latencies were found between the Ss in the two recall conditions. Thus, no apparent differences in mediators existed between the members of these two groups.

The discussion of the results now turns to an examination of response recall. The mean recall scores for each condition are plotted in Fig. 3. These data represent the three recall trials, the immediate and the delayed recall for the immediate condition, and the delayed recall trial for the delayed only group. Each of these recall trials will be discussed separately.

An analysis of variance of the immediate recall scores indicated that the type of pair was the only significant source of variance $[F(3,45)=19.21, p<.01]$. Subsequent analyses showed that recall was significantly higher for $\mathrm{CC}$ than for $\mathrm{CA}$ or $\mathrm{AC}$ pairs, which in turn were better recalled than AA pairs. These results reflect the usual pattern of reported effects of pair concreteness on recall performance (e.g., Yuille \& Paivio, 1968). Thus, in spite of the discovery and production time differences between images and verbal mediators, no recall differences as a function of mediator type

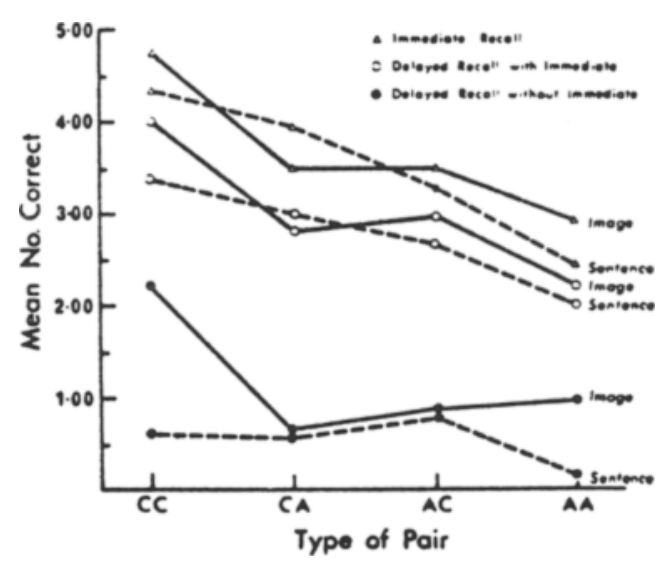

Fig. 3. Mean total response recall scores. 
appeared on the immediate recall trial. Similarly, concreteness affected the retrieval of sentence mediated pairs as much as it did recall of image mediated nouns. These findings are similar to those reported in all previous studies that have investigated concreteness, except the results reported by Paivio and Foth (1970).

Paivio and Foth (1970) reported three experiments in which instructions to form the two types of mediators were employed. In Experiment I, which required mediator production during the study trial, recall for imaginally mediated $\mathrm{CC}$ pairs was superior to verbally mediated recall, while the reverse was true for AA pairs, with verbal mediation being superior. It should be noted that this interaction effect was small relative to the main effect of type of pair. In the second experiment they report, which did not require production of mediators, this interaction did not emerge. The third experiment compared recall of AA pairs for Ss required to produce mediators and those who did not produce their mediators. The superiority of verbally linked pairs reported in their first experiment was replicated only when mediator production was required. They concluded that concreteness and mediation instructions interact only when mediator production is required. The present study required production but did not find the interaction. The only apparent reason for this difference between the two studies is the self-paced aspect of the present experiment. Apparently, the time limit for each pair $(15 \mathrm{sec})$ used by Paivio and Foth (1970) was detrimental to image formation for AA pairs.

The Ss that gave immediate recall each returned 7 days later for a second recall trial. Their mean performance scores, shown in Fig. 3, present a pattern which is very similar to their immediate recall. There was a decline in recall after 7 days $[F(1,15)=23.90$, $\mathrm{p}<.01]$, but otherwise the results were the same. The effect of pair type was significant $[F(3,45)=10.48$, $p<.01]$, with the ordering of recall the same as on the immediate trial: $\mathrm{CC}>\mathrm{CA}=\mathrm{AC}>\mathrm{AA}$.

These data indicate a continuing effect of pair concreteness on recall after a delay of 1 week. They support the conclusion by Yuille (1971) that concreteness effects are potent with delay, contrary to the study reported by Butter (1970). However, this conclusion will be modified after considering the delay only recall performance.

The recall scores for the delay only condition present a very different picture from that found with the other two recall trials. First, a main effect of type of mediator was obtained $[F(1,15)=8.21, p<.01]$, but no main effect of pair type. This is the reverse of the pattern for the other two recall trials. The mediator type effect was qualified by a significant interaction $[\mathrm{F}(3,45)=6.87$, $p<.01]$. The post hoc tests confirmed the following pattern. Response recall for image mediated pairs exceeded verbally mediated recall for $\mathrm{CC}$ and AA pairs, but recall was the same for both types of mediators for $\mathrm{CA}$ or $\mathrm{AC}$ pairs. The alternative view of this interaction is that pair concreteness had no effect on response recall for sentence mediated pairs. However, when images were used, $\mathrm{CC}$ recall exceeded that for the other three pair types.

To summarize these effects, if Ss do not have an immediate recall trial, and if they have used verbal mediators, their delayed recall performance will be unaffected by pair concreteness; in fact, recall will be too low to permit any pair differences. Thus, the lack of a concreteness effect on recall after a delay reported by Palermo (1970) may have been a result of his Ss using verbal as opposed to imaginal codes. Clearly, the long-term potency of concreteness in recall trials is a complex result of the type of recall experience (immediate vs no immediate) and the type of mediator used during the study trial.

The effects of an immediate recall experience are found in the comparison of the two delayed recall trials. The immediate group performed significantly better after 7 days than did the delay only group $[F(1,30)=$ $39.89, \mathrm{p}<.01]$. In fact, the immediate group averaged $340 \%$ higher recall than did the delayed condition. This finding is consistent with the effects of an immediate test on memory for prose reported by Rothkopf (1966) and represents a surprisingly potent effect of an immediate recall experience on subsequent recall ability in a paired-associate task. It seems that the practice of retrieving information after a study trial affects the subsequent retrieval of the response. This retrieval practice effect may reflect two effects of immediate recall on subsequent recall. It is possible that immediate retrieval allows $\mathrm{S}$ to improve storage for the pair. For example, alternative storage aids might be discovered at the time of recall. However, this author prefers to interpret the retrieval practice effect as an indication of two stages in the learning process. That is, in addition to the storage or acquisition of information, $\mathrm{S}$ must learn to retrieve. Perhaps learning how to retrieve newly acquired information is essential in guaranteeing subsequent recall. Note also that retrieval practice seems to differentially effect images and verbal mediators. Verbally mediated responses were more poorly recalled than were image mediated responses, when no immediate retrieval. practice was permitted. Before pursuing this point, however, the recall of the mediators themselves must be examined.

Mediators were scored as correctly or incorrectly recalled by two independent judges. A mediator was classified as correctly recalled if it was the same as or similar to the mediator produced during the study trial. The judges agreed on $94.5 \%$ of their initial classifications and were able to agree through discussion on the remainder.

The mean number of correct mediators for each condition are represented in Fig. 4. On the immediate recall trial, the type of pair affected mediator recall $[F(3,45)=23.33 . p<.01]$. More mediators were recalled when the stimuli were concrete $(\mathrm{CC}$ and $\mathrm{CA}$ 


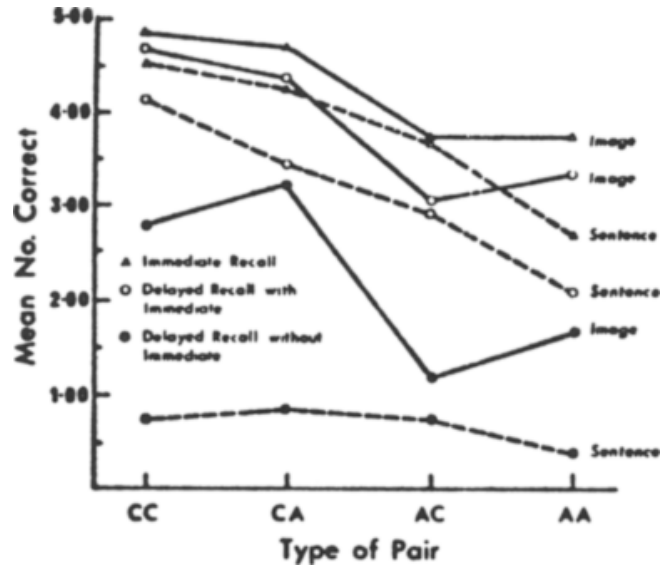

Fig. 4. Mean total mediator recall scores.

pairs) than when they were abstract (AC and AA pairs) regardless of mediator type. In addition, images were better recalled than were verbal links for all pair types $[F(1,15)=8.81, p<.01]$. This pattern of immediate mediator recall is quite different from that of immediate response recall. An explanation for this is presented below after the mediation recall for the two delay trials is examined.

After a 1-week delay, the immediate group showed exactly the same pattern of mediator recall as on the immediate trial. The effect of pair type was significant $[F(3,45)=18.35, p<.01]$, as was the difference between the two types of mediators $[F(1,15)=15.01$, $\mathrm{p}<.01]$. While the recall pattern was the same, overall performance was lower on the delayed recall trial $[F(1,15)=14.00, p<.01]$.

Once again, the results for the delay only group are very different from the delayed performance of the immediate Ss. First, as shown in Fig. 4, the superior recall of images over verbal mediators is much greater for the delay only condition $[F(1,15)=74.09, p<.01]$. While the type of pair was a significant source of variance $[F(3,45)=10.15, p<.01]$, it interacted with mediator type $[F(3,45)=8.20, p<.01]$. As confirmed by the post hoc tests, pair concreteness had no effect on sentence recall. With images, however, mediator recall was significantly higher for concrete stimulus pairs (CC and $\mathrm{CA}$ pairs) than for abstract stimulus pairs ( $\mathrm{AC}$ and AA pairs).

It is apparent that the retrieval practice effect found with response recall also affects mediator recall. Yet there are some interesting differences in the effects of immediate recall on subsequent response and mediator retrieval. The most important one is that the recall of images seems less affected by retrieval practice than does sentence recall. As an inspection of Fig. 4 will reveal, sentence recall is negligible without retrieval practice. Image recall, while lower without retrieval practice, does not suffer anywhere near the loss that is found with sentences. Thus, these results have revealed not only a surprising effect of an immediate recall trial on subsequent recall, but that this effect interacts with the type of storage employed for a pair.

The mediator recall and response recall results have revealed different effects of pair and mediator type on these two recall measures. With response recall, $\mathrm{CC}$ recall exceeds $\mathrm{CA}$ and $\mathrm{AC}$ recall, which in turn are superior to AA recall. The pattern for mediator retrieval, on the other hand, is that performance was equal for $\mathrm{CC}$ and $\mathrm{CA}$ pairs and superior to performance with $\mathrm{AC}$ and $\mathrm{AA}$ pairs. An additional difference is found in that response recall did not vary as a function of mediator type (except in the delay only condition), while mediator recall was superior for images on all three recall trials (especially the delay only condition). Finally, if Figs. 3 and 4 are compared, it will be seen that mediator recall generally exceeded response recall.

An explanation for these differences can be found in the hypothesized relationship between mediator retrieval and response retrieval. ${ }^{4}$ If we assume that with the presentation of a stimulus noun on a recall trial $S$ first retrieves his mediator and then uses the mediator to retrieve the response, then the differences between the two types of recall can be attributed to the latter decoding stage. That is, the data suggest that images are recalled better than verbal codes but that decoding the correct response from an image is more difficult than from a verbal mediator. This seems intuitively reasonable in that a sentence mediator usually contains the correct response noun. If the verbal mediator is retrieved, then the response term will be available. An image, alternatively, contains a nonverbal representation of a referent of the response noun. Since objects usually have multiple labels, there is a likelihood that $S$ may incorrectly decode his image and give the wrong response. This should be especially true when the response is an abstract noun, where the relationship between noun and referent is less direct.

In order to explore this proposed relationship between mediator and response recall, the number of decoding errors for each condition was examined. A decoding error was defined as occurring when $S$ correctly recalled his mediator but did not recall the correct response (making either an intrusion error or an omission). Figure 5 presents the means of these errors for each condition. The pattern of the errors is very striking indeed. On each of the three recall trials, more decoding errors are associated with images than with sentence mediators $[F(1,15) \geqslant 7.85, p<.01]$. While the effect of pair type was significant in each case $[F(3,45) \geqslant 5.56, p<.01]$, each analysis also revealed a significant interaction $[F(3,45) \geqslant 8.72, p<.01]$. These interaction effects were the same on each of the three trials: decoding errors to images exceeded sentence-associated errors only when the response was abstract (CA and AA pairs). In other words, with concrete responses, both image and verbally mediated pairs have equally low decoding errors. However, with 
abstract responses, the proposed decoding difficulty associated with images is confirmed by the significantly higher number of decoding errors.

Examples of the types of intrusion errors that Ss make with image mediated pairs suggest that they are errors in only the narrowest sense of the word. For example, with the pair CIRCLE-PERSON, Ss frequently drew a circle with a stick man inside. On the recall trial, they would reproduce the mediator but write the response word MAN. While this response is scored as an error in a PA learning experiment, it would not be incorrect "in real life." Synonym responses are acceptable outside of the laboratory. Thus, experiments such as the present one probably underestimate the potency of nonverbal coding because of the strict scoring procedures.

The recall latencies add some relevant information to the discussion of the recall process. Three types of latencies could be generated from these data: (1) median latencies when both the mediator and response were correctly recalled; (2) median latencies when only the mediator was correct (decoding error); and (3) latencies when both mediator and response were incorrect. Table 2 provides the means of each of these latencies for the three recall trials. These entries are group means of the medians generated for each $\mathrm{S}$ and are based on widely disparate Ns.

The variability in the number of observations available for the recall latencies restricted the number of parametric tests that could be performed. Sufficiently large Ns were available only for comparisons within a particular latency type. Analyses of variance were conducted on the both correct (response and mediator) latencies for the immediate group, separately for each recall trial. In addition, latencies for pairs for which both mediator and response were incorrect were analyzed for the delay group. All three analyses indicated no effect of mediator type. In other words, images and verbal mediators cannot be discriminated on the basis of retrieval time. However, correct mediators and responses

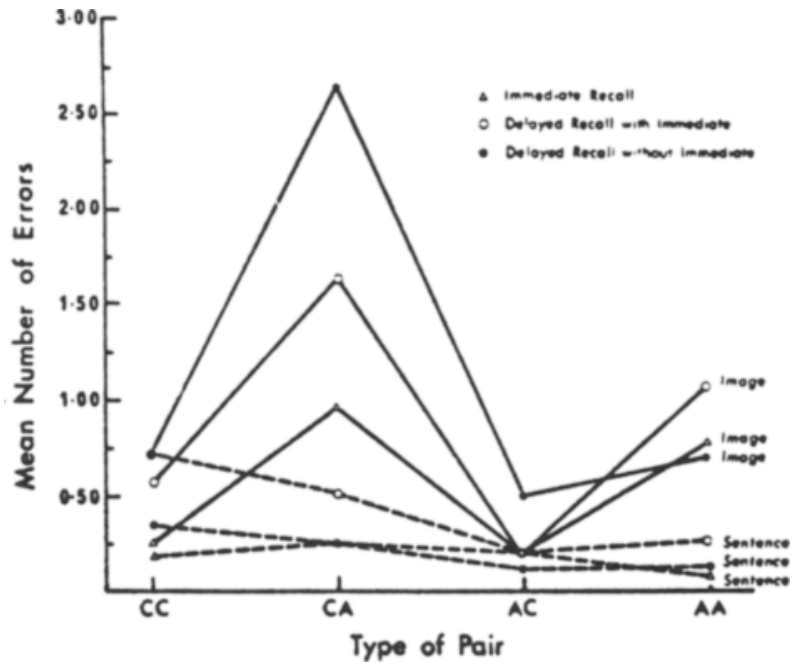

Fig. 5. Mean number of decoding errors.

required significantly longer to recall for AA pairs than for the other three pair types $[F(3,36)=7.85, p<.01]$.

Only nonparametric tests could be made between the different latency types. These tests involved comparing the response latencies for pairs for which both mediator and response were correct with the latencies for pairs for which only the mediator was correct within an experimental condition. Four out of five sign tests proved recall to be more rapid when both mediator and response were correct $(Z \geqslant 2.22)$. These data suggest that after mediator retrieval, $\mathrm{S}$ takes longer response time to attempt to retrieve the response. In addition, the mediator's only correct latencies were significantly shorter than the both wrong latencies in two out of three sign tests $(Z \geqslant 2.02)$. Finally, the latencies when both mediator and response were correct were significantly shorter than when both were wrong in seven out of eight sign tests $(Z \geqslant 2.02)$. In summary, there is a general trend for most rapid responding when both mediator and response are correct, somewhat

Table 1

Means of Recall Latencies

\begin{tabular}{|c|c|c|c|c|c|c|c|c|}
\hline \multirow{2}{*}{$\begin{array}{l}\text { Type of Pair } \\
\text { Type of Mediation }\end{array}$} & \multicolumn{2}{|c|}{$\mathrm{CC}$} & \multicolumn{2}{|c|}{$\mathrm{CA}$} & \multicolumn{2}{|c|}{$\mathrm{AC}$} & \multicolumn{2}{|c|}{ AA } \\
\hline & Image & $\begin{array}{l}\text { Sen- } \\
\text { tence }\end{array}$ & Image & $\begin{array}{l}\text { Sen- } \\
\text { tence }\end{array}$ & Image & $\begin{array}{l}\text { Sen- } \\
\text { tence }\end{array}$ & Image & $\begin{array}{l}\text { Sen- } \\
\text { tence }\end{array}$ \\
\hline \multicolumn{9}{|l|}{ Immediate Recall } \\
\hline Response and Mediator Correct & 3.65 & 4.10 & 5.27 & 4.74 & 4.77 & 6.01 & 7.20 & 7.64 \\
\hline Mediator Only Correct (Decode Error) & 6.34 & 5.56 & 23.18 & 14.83 & 10.08 & 10.98 & 17.48 & 14.66 \\
\hline Both Wrong & 10.36 & 7.89 & 24.62 & 27.04 & 22.53 & 22.13 & 25.54 & 28.28 \\
\hline \multicolumn{9}{|l|}{ Delayed Recall with Immediate } \\
\hline Response and Mediator Correct & 4.48 & 4.56 & 6.68 & 4.07 & 5.28 & 5.24 & 11.13 & 6.70 \\
\hline Mediator Only Correct (Decode Error) & 7.01 & 7.59 & 10.83 & 9.13 & 14.83 & 7.24 & 9.40 & 12.36 \\
\hline Both Wrong & 16.06 & 18.39 & 17.66 & 10.87 & 17.05 & 14.16 & 14.62 & 13.63 \\
\hline \multicolumn{9}{|l|}{ Delayed Recall without Immediate } \\
\hline Response and Mediator Correct & 14.51 & 6.00 & 6.54 & 4.31 & 10.56 & 6.13 & 12.15 & 8.78 \\
\hline Mediator Only Correct (Decode Error) & 12.53 & 12.33 & 9.24 & 12.51 & 15.33 & 15.88 & 21.83 & 11.40 \\
\hline Both Wrong & 25.17 & 24.40 & 22.89 & 21.49 & 23.26 & 20.09 & 24.21 & 22.12 \\
\hline
\end{tabular}


Table 2

Percentage of Recalled Mediators Incorrectly Decoded

\begin{tabular}{lrrrrrrrr}
\hline & \multicolumn{10}{c}{ Type of Pair } \\
\cline { 2 - 9 } \multicolumn{1}{c}{ Type of Mediator } & Image & Sentence & Image & Sentence & Image & Sentence & Image & Sentence \\
\hline Immediate & 5.11 & 4.23 & 20.27 & 5.88 & 5.08 & 5.17 & 20.34 & 2.33 \\
Delayed with Immediate & 12.16 & 16.67 & 37.68 & 14.55 & 6.12 & 6.52 & 32.08 & 12.12 \\
Delayed without Immediate & 25.00 & $\mathbf{( 4 5 . 4 5 )}$ & 80.77 & $133.33)$ & $(42.11)$ & $(18.18)$ & 42.31 & $(40.00)$ \\
\hline
\end{tabular}

Note-Percentages in parentheses are all based on less than 20 pairs and mav not be representative of these conditions.

longer response times when only the mediator is correct, and longer latencies when both mediator and response are incorrect.

The final aspect of these results that requires discussion is the relationship between discovery and production latencies and recall. Since images required significantly longer to discover and produce than did sentences, the superior recall of the former might simply be a function of study time. A statistical examination of this relationship did not support this hypothesis. Within each pair type, the median discovery latencies for correctly and incorrectly recalled mediators were compared, separately for images and sentences. None of these differences were significant, the values of the $t$ tests varying from .01 to 1.04 . This pattern was exactly the same for production latencies and was the same when related to response recall rather than to mediator recall. Thus, it is impossible to predict recall of a sentence or of an image from its discovery or production latency.

\section{SUMMARY AND CONCLUSIONS}

Before presenting the major conclusions of this study, a brief summary of the major findings is given. The discovery and production of image mediators is a direct function of pair concreteness. Not only does production and discovery take longer for more abstract pairs, but it also appears that images mediating these pairs may be qualitatively different (more complex) than images generated for concrete pairs. Recall of images is affected by stimulus concreteness, while the decoding of an image to produce the response is affected by response noun concreteness. Finally, images are better recalled than their verbal counterparts and are less affected by lack of retrieval practice than are verbal mediators.

The discovery and production of sentence mediators is unaffected by pair concreteness. However, recall of verbal mediators is affected by stimulus concreteness. Few decoding errors are associated with verbally mediated recall, and these errors are not related to response concreteness. While the recall of sentence mediators is generally poorer than image recall, recall of verbal mediators is also more affected by lack of retrieval practice.

These results have revealed a variety of new information about coding, storage, and retrieval processes in associative learning. First, the distinction between verbal and nonverbal coding has been expanded. From previous experiments (e.g., Yuille \& Paivio, 1968), it was known that image discovery varied with pair concreteness, while the discovery of verbal mediators did not. The results of the present study add that the characteristics of images also vary with pair concreteness. The longer production times for images linking abstract nouns is interpreted as indicating more complexity and detail in images to more abstract material. It is possible that the longer image discovery times to abstract pairs reflect the greater time required to generate a complex image. Intuitively, however, it is suspected that the associative processes linking abstract nouns to referents require longer than those linking referents to their concrete labels.

A second important consequence of these data is their capacity to resolve some empirical inconsistencies. A variety of disparate results have been reported relating the type of mediator and pair concreteness to response recall. Generally, no difference between the recall of image and verbal mediated pairs has been reported (e.g., Yuille \& Paivio, 1968). Yet the different characteristics of the two mediator types in relationship to concreteness (e.g., discovery time) has made this lack of a recall difference somewhat difficult to explain. The present results suggest that different processes are involved in retrieval of each mediator type. First, the more concrete the stimulus member, the easier it is to recall an image. Presumably this, once again, reflects the more direct (and rapid) associative processes between referents and concrete nouns. Once retrieved, the image must be decoded, i.e., the appropriate label must be retrieved for the content of the image. As noted previously, the ease of decoding will be a function of the specificity of the image and the abstractness of the label. The less specific the content of an image is, or the less direct its relationship to the response word, the more likely it is that a decoding error will occur.

Verbal mediator retrieval, alternatively, should not vary as a function of concreteness. The fact that it does suggests either that some nonverbal coding is spontaneously generated by verbal codes or that concrete and abstract nouns are differentially stored in the verbal system. No preference is given here for either of these alternatives, although concreteness clearly has no effect on sentence retrieval after a 1 -week delay when no retrieval practice occurs.

This latter result also indicates the reason for the 
discrepancy between the results reported by Butter (1970) and Palermo (1970) and those of Yuille (1971). The former investigators reported a reduced or reversed effect of concreteness on recall after a delay, while the latter study did not report this. The present study indicates that the long-term effect of concreteness on recall depends on (1) the opportunity for retrieval practice; and (2) the type of code Ss use. Depending on these factors, it is possible to produce strong, reduced, or no concreteness effects on delayed recall.

In addition to the above differences between the two codes, it is apparent that images are better associative codes than are sentences. It has been reported in the past that memory for pictures is superior to memory for sentences (Shepard, 1967). Apparently, this is also true when $S$ generates the two types of representation. However, there exists no evidence which would permit an interpretation of this difference. The reason for a superior retrieval capacity in one memory modality over another remains a mystery.

The most important finding of this study is the interaction of retrieval practice and type of mediator in determining delayed recall. As noted above, the main effect of immediate recall conforms to that reported earlier (e.g., Rothkopf \& Bisbicos, 1967). Two explanations for the interaction are considered below.

It is possible that an immediate recall trial acts as an additional study trial. That is, when $\mathbf{S}$ correctly recalls a response noun, the noun pair is available for further study. The idea that recall can improve storage is not a novel one (cf. Kintch, 1970). In particular, this proposal is similar to one included in Adams and Bray's (1970) closed loop theory of PA learning. They hypothesized that an SR perceptual trace and an SR memory trace are established for each pair. Once a strong perceptual trace is acquired for a particular pair, subsequent retrieval of the response serves as an occasion for reinforcement of the memory trace. While this hypothesis is difficult to test here, some of the present results do not seem consistent with an SR reinforcement idea. For example, because of the self-paced nature of the study trial, original learning was quite high, and it is difficult to understand why one immediate test trial should so strongly reinforce a memory trace. Furthermore, it is not apparent that the Adams and Bray (1970) model would predict a differential effect of immediate recall on decoding errors. Finally, since the model does not postulate different coding systems, it would not predict the interaction of Type of Mediator by Retrieval Practice in delayed recall.

An alternative explanation for the present results involves a more cognitive interpretation of PA learning. This hypothesis is concerned with the effects of recall on storage, as is the above model; however, two stages are postulated for the PA learning situation. First, it is assumed that $\mathrm{S}$ must discover and store a mediator during the study trial. Learning to retrieve and to decode the mediator is proposed as the second stage of learning.
Consequently, an immediate study trial permits $S$ to determine the ease of retrieval of his mediators and to determine how readily he can decode them. It is assumed that an immediate test is better than a delayed test for this purpose, because extraexperimental sources of interference are at a minimum. Now, if $\mathbf{S}$ has some difficulty retrieving a mediator but eventually does so successfully, this may encourage him to find a new code for that pair. Alternatively, $\mathrm{S}$ might retrieve a mediator with ease but find some difficulty decoding it. This should encourage $S$ to elaborate the code to prevent decoding problems, or perhaps to generate a new code. In other words, the immediate test trial is a vehicle for $S$ to check ease of mediator retrieval and ease of decoding.

The above hypothesis is similar to one proposed by Prytulak (1971) for the learning of CVCs. He suggested that Ss store a natural language mediator (NLM) and a transformation tag for each CVC. The recall of a CVC involves retrieval of the NLM and the tag. The tag permits $S$ to apply the appropriate transformation to reinstate the original $\mathrm{CVC}$. Relating this to the present context, generating an NLM is comparable to discovery of a mediator and applying a transformation (or set of transformations) is equivalent to decoding a mediator. In fact, it seems reasonable to equate the present hypothesis with that proposed by Prytulak (1971). Montague (1972) has suggested that the Prytulak (1971) analysis is applicable to the effects of noun concreteness.

This two-stage hypothesis of PA learning can be used to reinterpret some of the present findings. For example, since image mediator recall does not suffer as much from lack of retrieval practice as do verbal mediators, it is appropriate to propose that images are more likely to be appropriately coded during the study trial than are verbal codes. That is, ease of retrieval of images seems better than ease of verbal mediator retrieval. Thus, in terms of mediator recall, an immediate test is more valuable for verbally coded and should lead to improvement of the codes for those pairs.

On the other hand, visual codes seem more susceptible to decoding problems, particularly if the pair contains an abstract response noun. Consequently, an immediate test should reduce subsequent decoding errors for image mediated pairs, at least when the response is abstract. This deduction can be evaluated by examining the percentage of correctly recalled mediators that were decoded incorrectly. Table 2 presents the percentage of mediators decoded incorrectly for each of the three recall trials. For the immediate test, the results conform with the hypothesis. Images have a higher proportion of decoding errors than do verbai mediators for $\mathrm{CA}$ and $\mathrm{AA}$ pairs, but not for concrete response pairs. This pattern remains the same for the immediate test Ss after 1 week, although the image/sentence difference is reduced. More important, after a 1-week delay, the Ss with no immediate test show a much higher proportion of decoding errors with images than do those $S s$ given retrieval practice. For example, with $\mathrm{CA}$ pairs and no 
immediate test, $80.77 \%$ of images are incorrectly decoded, but with an immediate test only $37.68 \%$ of images have decoding errors. These data are consistent with the notion that an immediate test improves subsequent decoding of visual mediators. Unfortunately, too few verbal codes were recalled in the no immediate test group (11 or less per pair type), and the effects of retrieval practice on sentence decoding cannot be determined.

To summarize, these results (as well as those of Prytulak, 1971) are consistent with a two-stage hypothesis of PA learning. Furthermore, it appears that an immediate recall trial may affect subsequent retrieval (more than subsequent decoding) of verbal codes, while the reverse is true for nonverbal codes. The similarity of the present interpretation to that of Prytulak (1971) suggests that future research in this field should concentrate on generating a transformational grammar for visual and verbal codes, similar to the transformation stack idea presented by Prytulak (1971).

\section{REFERENCES}

Adams, J. A., \& Bray, N. W. A closed-loop theory of paired-associate verbal learning. Psychological Review, 1970, $77,385-405$.

Bower, G. H. Mental imagery and associative learning. In $\mathbf{L}$. Gregg (Ed.), Cognition in learning and memory. New York: Wiley, 1972.

Bruning, R. H. Effects 'of review and test-like events within the learning of prose material. Journal of Educational Psychology, 1968, 59, 16-19.

Bugelski, B. R. Presentation time, total time, and mediation in paired-associate learning. Journal of Experimental Psychology, $1962,63,409-412$.

Butter, M. J. Differential recall of paired associates as a function of arousal and concreteness-imagery levels. Journal of Experimental Psychology, 1970, 84, 252-256.

Frase, L. T. Learning from prose material: Length of passage, knowledge of results, and position of questions. Journal of Educational Psychology, 1967, 58, 266-272.

Gates, A. I. Recitation as a factor in memorizing. Archives of Psychology, 1917, 26, No. 1.

Humphreys, M. S., \& Yuille, J. C. Errors as a function of noun concreteness. Canadian Journal of Psychology, in press.

Kiess, H. O., \& Montague, W. E. Natural language mediators in paired associate learning. Psychonomic Science, 1965, 3, $549-550$

Kintch, W. Learning, memory and conceptual processes. New York: Wiley, 1970.

Martin, C. L., Cox, D. L., \& Boersma, F. J. The role of associative strategies in the acquisition of PA material: An alternative approach to meaningfulness. Psychonomic Science, $1965,3,463-464$.

Montague, W. E. Elaborative strategies in verbal learning and memory. In G. H. Bower (Ed.), The psychology of learning and motivation. Vol. 6. New York: Academic Press, 1972.

Montague, W. E., Adams, J. A., \& Kiess, H. O. Forgetting and natural language mediation. Journal of Experimental Psychology, 1966, 72, 829-833.

Paivio, A. Imagery and verbal processes. New York: Holt, Rinehart \& Winston, 1971

Paivio, A., \& Foth, D. Imaginal and verbal mediators and noun concreteness in paired-associate learning: The elusive interaction. Journal of Verbal Learning \& Verbal Behavior, $1970,9,384-390$.

Paivio, A.. \& Yuille, J. C. Changes in associative strategies and paired-associate learning over trials as a function of word imagery and type of learning set. Journal of Experimental Psychology. 1969.79, $458-463$

Paivio. A. Yuille. J. C.. \& Madigan. S. A. Concreteness, imagery and meaningfulness values for 925 nouns. Journal of Experimental Psychology. 1968. 76(Monogr. Suppl. 1. Pt. 2).

Pairio. A.. Yuille. J. C.. \& Smythe. P. C. Stimulus and response abstractness. imagery and meaningfulness and reported mediators in paired-associate learning. Canadian Journal of Psychology. 1966, 20, 362-377

Palermo. D. S. Imagery in children's learning: Discussion. Psychological Bulletin. 1970. 73, 415-421

Prytulak, L. S. Natural language mediation. Cognitive Psychology. 1971. 2. 1-56.

Rothkopf. E. Z. Learning from written materials: An exploration of the control of inspection behavior by test-like events. American Educational Research Journal, 1966. 3. 241.249

Rothkopf, E. Z., \& Bisbicos. E. Selective facilitative effects of interspersed questions on learning from written materials. Journal of Educational Psychology, 1967, 58, 56-61.

Schnorr, J. A.. \& Atkinson. R. C. Repetition versus imagery instructions in the short- and long-term retention of paired associates. Psychonomic Science. 1969, 15, 183-184.

Shepard. R. N. Recognition memory for words, sentences and pictures. Journal of Verbal Learning \& Verbal Behavior, 1967, 6. 156-163

Yuille, J. C. Does the concreteness effect reverse with delay? Journal of Experimental Psychology, 1971, 88, 147-148.

Yuille, J. C., \& Paivio, A. Latency of imaginal and verbal mediators as a function of stimulus and response concreteness-imagery. Journal of Experimental Psychology, $1967,75,540-544$.

Yuille. J. C., \& Paivio. A. Imagery and verbal mediation instructions in paired-associate learning. Journal of Experimental Psychoiogy, 1968, 78, 436-441.

\section{NOTES}

1. The term mediation is used in this report to refer to elaboration processes that create an interaction or association between the members of a pair. While rote learning is mediated to the extent that $S$ must recognize and label nouns in order to repeat them, it is assumed that this represents a basic, nonelaborative level of processing and is considered as nonmediated learning. An excellent definition of rote learning, consistent with that employed in the present article, is given by Prytulak (1971, p. 1).

2. Paivio (1971) has suggested that storage of information in the visual system may be parallel in form, while verbal storage is hypothesized as being of a sequential character. Inferior verbal memory would then be a consequence of the fact that the retrieval of each link in sequential verbal storage is dependent on retrieval of earlier links, while such interdependency between components does not exist in parallel visual storage.

3. Due to the important implications of the results reported in this study, it should be noted that an earlier pilot investigation involving $40 \mathrm{Ss}$ was conducted. The pattern of the reported recall results is the same as that found in the pilot investigation. The data from the latter are not reported because of procedural differences between it and the major investigation. In particular, the latencies were not obtained in the pilot investigation.

4. Although mediators were recalled without recall of the appropriate response (decoding errors), the reverse was a rare occurrence. The number of correct responses without correct mediator recall was $1(0.2 \%)$ on the immediate test, $8(2.1 \%)$ for the same group 1 week later, and $10(9.2 \%)$ for the delay only group.

(Received for publication January 2, 1973: revision received March 3.1973.) 